\title{
Axisymmetric Spindown Dynamics of Hurricane-like Vortices
}

\author{
Michael T. Montgomery, Holly D. Snell, and Zonguun Yang \\ Department of Atmospheric Science, Colorado State University, Fort Collins, Colorado
}

(Manuscript received 18 October 1999, in final form 23 May 2000)

\begin{abstract}
This work examines the spindown problem of hurricane-like vortices subject to a quadratic drag law in the surface layer. Since intense hurricanes over the open ocean are approximately axisymmetric due in part to Rossby elasticity and axisymmetrization processes that tend to keep the vortex erect and circular, the axisymmetric spindown problem serves as a useful benchmark. As a basis for the numerical experiments presented, the essential results of Eliassen and Lystad's balanced spindown-spinup theory are reviewed first. The theory is then tested with an axisymmetric Navier-Stokes numerical model. The numerical experiments broadly confirm the theoretical predictions for a range of vortex heights, maximum tangential wind speeds, constant and variable drag coefficients, and vortex sizes considered relevant for tropical storm and hurricane strength vortices. But unlike the monotonic decay of the swirling flow predicted by theory, the numerical simulations reveal a temporary spinup of the tangential winds in the boundary layer before the demise of the vortex.

The theory is shown to furnish a consistent description of the weakening phase of two hurricanes observed by research aircraft. Despite the idealizations employed to yield a tractable model, the theory appears useful in elucidating weakening episodes of hurricanes not associated with strong asymmetries.
\end{abstract}

\section{Introduction}

Hurricanes are destructive tropical storms characterized by intense swirling winds and torrential rainfall. When penetrative convection in the eyewall and spiral bands becomes suppressed, the vortex loses its ability to gather the angular momentum necessary to maintain itself against frictional dissipation and will subsequently decay. Many previous studies have been directed at understanding axisymmetric hurricane intensification and quasi-steady equilibrium (e.g., Ooyama 1969; Rosenthal 1978; Willoughby et al. 1984; Rotunno and Emanuel 1987), but comparatively little work has been directed at understanding the dynamics of spindown for hurricane strength vortices. Since intense storms over the open ocean are approximately axisymmetric (Shapiro and Montgomery 1993, Fig. 1) due in part to Rossby elasticity and axisymmetrization processes that tend to keep the vortex erect and circular (Reasor and Montgomery 2000, manuscript submitted to J. Atmos. Sci.; and references therein), the axisymmetric spindown problem serves as a useful benchmark and will be the focus of this paper.

The conceptual model for vortex spindown is well known. Above the boundary layer, an approximate bal-

Corresponding author address: Dr. Michael T. Montgomery, Dept. of Atmospheric Science, Colorado State University, Fort Collins, CO 80523-1371.

E-mail: mtm@charney.atmos.colostate.edu ance exists between centrifugal, Coriolis, and pressure gradient forces. Friction near the sea surface reduces the tangential wind relative to its bulk value, and since the pressure field is approximately constant with height in the boundary layer an uncompensated radial pressure gradient drives a radial inflow toward the center that causes divergent flow above the boundary layer. Assuming fluid parcels conserve their angular momentum above the boundary layer, the divergent flow implies a decay of the tangential winds in the interior flow signifying the spindown of the vortex. Surprisingly little is known, however, about how spindown depends on the given flow parameters (e.g., maximum tangential wind speed, radius of maximum tangential wind, fluid depth, surface drag) for a hurricane strength vortex subject to a quadratic drag law in the surface layer and to what extent the surface winds intensify before the demise of the vortex.

The spindown problem for geophysical vortices appears to have been investigated first by Eliassen (1971). Both laminar and turbulent surface boundary conditions were considered and solid body rotation was assumed in the interior fluid. For the case of a laminar boundary layer the principal results were in accord with the pioneering work by Greenspan and Howard (1963). Specifically, the temporal decay of the interior tangential winds is exponential and the (upward) vertical velocity at top of the Ekman layer is maximum at the center and approximately constant inside the radius of maximum tangential winds (RMW) of the vortex. For the case of 


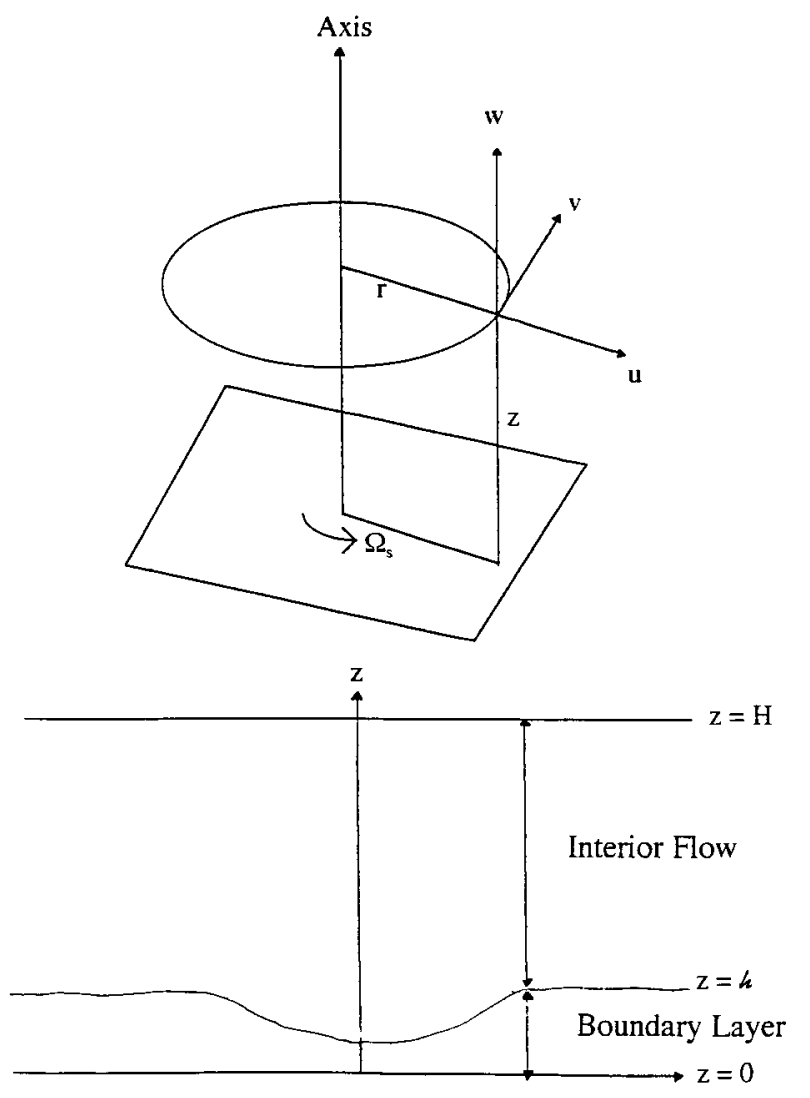

FIG. 1. (a) The inertial coordinate system where $r$ denotes radius, $\lambda$ the azimuth, and $z$ the height above the lower surface. (b) Model setup of a homogeneous and incompressible boundary layer and interior fluid confined between two parallel horizontal plane surfaces rotating with an angular frequency $\Omega_{s}$.

a turbulent boundary layer, Eliassen's theory predicts an algebraic temporal decay and a vertical velocity at the top of the Ekman layer which vanishes at the center and increases linearly with radius inside the RMW of the vortex. Eliassen and Lystad (1977, hereafter EL) later extended this work to account for differential rotation in the swirling flow. ${ }^{1}$

\footnotetext{
${ }^{1}$ Although prior work by Rosenthal (1962) and Smith (1968) anticipated the vertical structure of the hurricane Ekman layer, and Ooyama's (1969) model contained the prognostic boundary layer equations that were later developed in a different manner by Eliassen (1971) and EL for a homogeneous (neutrally stratified) fluid, Eliassen was the first to combine these two approaches for the purpose of examining the axisymmetric spindown-spinup problem for geophysical vortices. Independent work on the spindown problem using a variant of an Oseen method of linearization was carried out by McWilliams (1971) using a constant eddy viscosity and a no-slip boundary condition. A summary of some of McWilliams's results appeared in Carrier (1971). Subsequently, others have considered the spindown/spinup problem (Weidman 1976; Wu and Blumen 1982; Kloosterziel and van Heijst 1992; Dolzhanskii et al. 1992 and references therein) but in other contexts and evidently unaware of Eliassen (1971) and EL.
}

Eliassen and Lystad developed an axisymmetric balance theory that neglects inertial oscillations. The theory predicts the evolution of the angular velocity, the transverse streamfunction, the boundary layer depth and the half-life time (the time required to reduce the angular velocity by half) for the spindown of a geophysical vortex subject to a quadratic drag law in the surface layer. Eliassen and Lystad's theory was formulated in an inertial (nonrotating) coordinate system and rests on the assumption that the flow evolves close to a state of cyclostrophic balance throughout the fluid. The strongest vortex EL used, however, was $10 \mathrm{~m} \mathrm{~s}^{-1}$, corresponding to a Rossby number of 20 at the latitude considered. The deficiency of the theory is that the neglected noncyclostrophic terms in the boundary layer may become significant at higher swirl speeds. It is not known whether the theory makes accurate predictions for hurricane strength (i.e., maximum tangential winds $>33 \mathrm{~m} \mathrm{~s}^{-1}$ ) vortices. One of the goals of this paper is to assess the validity of the theory for hurricane strength vortices over the open ocean. This is believed a necessary first step toward understanding the more complex spindown problem of a landfalling hurricane (e.g., Tuleya et al. 1984; Kurihara 1985; Wakimoto and Black 1994; Willoughby and Black 1996; Kaplan and DeMaria 1995; Wurman and Winslow 1998), or a hurricane undergoing extratropical transition (Jones and Thorncroft 2000).

The outline of this paper is as follows. Eliassen and Lystad's theory is reviewed in section 2. The axisymmetric Navier-Stokes model used to test the theory for tropical storm and hurricane strength vortices is presented in section 3. Section 4 presents the benchmark experiments for vortices of tropical storm strength (19 $\left.\mathrm{m} \mathrm{s}^{-1}\right)$ and hurricane strength $\left(38 \mathrm{~m} \mathrm{~s}^{-1}\right)$ and compares the results against the theory. Sensitivity experiments are carried out in section 5. The theory is then applied to two observed hurricanes in section 6. Section 7 gives the conclusions.

\section{Review of Eliassen and Lystad's (1977) theory}

The theory is formulated in an inertial (nonrotating) system of cylindrical polar coordinates, where $r$ denotes radius, $\lambda$ the azimuth and $z$ the height above the lower surface (see Fig. 1a for illustration). The motion is assumed axisymmetric with respect to the axis of rotation (i.e., $\partial / \partial \lambda=0$ ) and the fluid is assumed to be homogeneous (neutrally stratified) and incompressible. The fluid is confined between two rigid horizontal planes at $z=0$ and $z=H$ that rotate at a constant angular velocity $\Omega_{s}$ (see Fig. 1b for illustration). Finally, the interior and boundary layer flow are assumed to evolve near a state of cyclostrophic balance.

The theory focuses on the balanced ("slow") evolution of the vortex caused by the toroidal circulation $(u, w)$ forced by the Ekman layer. The "high-frequency" toroidal (inertial) oscillations are eliminated by neglect- 
ing the material derivative terms in the radial and vertical momentum equations. These approximations are valid, respectively, provided $u \ll v$ (Willoughby 1979) and the square of the depth is small compared to the square of the diameter of the vortex core. The resulting radial and tangential momentum equations simplify, respectively, to

$$
\begin{aligned}
\psi_{z z z} & =\frac{m^{2}-M^{2}}{K r^{2}} \text { and } \\
m_{t} & =\frac{1}{r}\left(m_{r} \psi_{z}-m_{z} \psi_{r}\right)+K m_{z z},
\end{aligned}
$$

where subscripts denote partial derivatives, $t$ is time, $\psi(r, z, t)$ the streamfunction for the transverse flow in the $r-z$ plane from which $u=\psi_{z} / r$ and $W=\psi_{r} / r$, $m(r, z, t)$ the absolute angular momentum of the fluid, $M(r, t)$ the absolute angular momentum of the interior fluid above the Ekman layer, and $K$ the constant eddy diffusivity. Eliassen and Lystad integrated these equations with free-slip boundary conditions on the upper lid and semislip boundary conditions on the lower lid (see EL or section $3 \mathrm{~b}$ for details). Integrations were initialized with the nondimensional angular momentum

$$
m_{0}(r)=\left[1+\omega_{0}(r)\right] r^{2},
$$

where

$$
\omega_{0}(r)=\frac{\mathrm{Ro}}{1+(r / \mathrm{RMW})^{2}}
$$

is the specified profile of the initial nondimensional angular velocity of the fluid relative to the rotating surfaces. The Rossby number, Ro, defined here by $\omega_{0}(r=$ $0) / \Omega_{s}$, represents the ratio of the angular velocity at the vortex center to the angular velocity of the boundary surfaces (i.e., $\Omega_{s}=f / 2$, where $f$ is the Coriolis parameter). The angular velocity used by EL was $\Omega_{s}=1.9$ $\times 10^{-5} \mathrm{~s}^{-1}$, corresponding to a latitude of $15^{\circ}$. Simulations were performed with an RMW of $50 \mathrm{~km}$; Rossby numbers of $1,5,10$, and 20; and drag coefficients of $2.0 \times 10^{-3}, 6 \times 10^{-3}, 2.0 \times 10^{-2}$, and $2.0 \times 10^{-1}$. Note that a Rossby number of 20 corresponds to a maximum tangential velocity of only $10 \mathrm{~m} \mathrm{~s}^{-1}$. The integrations with these values yielded predictions for the boundary layer formation time; boundary layer depth; hodographs of the Ekman spiral; and the evolution of $m, \psi$, and the interior rotation rate $\Omega$.

The numerical results were confirmed with a heuristic theory that becomes asymptotically valid in the limit of a small drag coefficient $\left(C_{D} \leq 0.02\right)$. The physical assumptions that underpin the heuristic theory are that $m$ $=M$ and $\psi_{z z z}=0$ in the interior flow, $m$ deviates slightly from $M$ in the Ekman layer, and that vertical advection is everywhere small compared to the radial advection of angular momentum. The interior rotation rate predicted by the heuristic theory is given by

$$
\frac{1}{|\Omega|}=\frac{1}{\left|\Omega_{0}\right|}+\frac{\chi^{2} C_{D} r t}{H-h}, \quad\left(\text { with } \Omega \Omega_{0}>0\right),
$$

where $\Omega=\Omega(r, t)$ is the angular velocity of the interior fluid relative to the horizontal boundary surfaces, $\Omega_{0}=$ $\Omega_{0}(r)$ the initial relative angular velocity, $\chi$ the reduction factor, $C_{D}$ the drag coefficient, $t$ the time, $H$ the total fluid depth, and $h(r, t)$ the boundary layer height. The reduction factor is defined by EL as the ratio of tangential winds at the top of the surface layer to the tangential winds at the top of the Ekman layer. In practice, the top of the main inflow layer is used instead of the Ekman layer depth. The time required to reduce $\left|\Omega_{0}\right|$ by a factor of 2 , called the half-life, $t_{\text {half }}$, is given by

$$
t_{\text {half }}=\frac{H-h}{\chi^{2} C_{D} r\left|\Omega_{0}\right|}=\frac{H-h}{\chi^{2} C_{D}|\tilde{v}|},
$$

where $\tilde{v}$ is the initial relative tangential velocity. According to (6), the half-life at radius $r$ is proportional to the depth $(H-h)$ of the interior fluid and inversely proportional to the relative tangential velocity $(\tilde{\boldsymbol{v}})$ and the drag coefficient $\left(C_{D}\right)$. The heuristic theory predicts an algebraic temporal decay for a turbulent boundary layer. The boundary layer formation time, the boundary layer depth, and the net radial volume flux are also predicted by the theory (see EL for details). Equations (5) and (6) will be used as a basis for comparing the theory against the unfiltered equations.

\section{The axisymmetric Navier-Stokes model}

\section{a. Governing equations}

In accord with the simplified theory, the NavierStokes (NS) model is formulated in the inertial cylindrical polar coordinate system of Fig. 1. The unfiltered, nondimensional Navier-Stokes equations for axisymmetric motions are given by

$$
\begin{aligned}
m_{t}+\frac{1}{r} J(\psi, m) & =r\left(\frac{1}{r} m_{r}\right)_{r}+m_{z z}, \\
\eta_{t}+\frac{1}{r} J(\psi, \eta)+\frac{\eta}{r^{2}} \psi_{z}-\frac{2 m}{r^{3}} m_{z} & =\left[\frac{1}{r}(r \eta)_{r}\right]_{r}+\eta_{z z}, \\
-\left(\frac{1}{r} \psi_{r}\right)_{r}-\frac{1}{r} \psi_{z z} & =\eta .
\end{aligned}
$$

Equations (7)-(9) denote, respectively, the angular momentum equation, the equation for the evolution of azimuthal vorticity, and the invertibility relation connecting azimuthal vorticity $(\eta)$ with the streamfunction $(\psi)$ for the transverse circulation. In (7) and (8) $J$ is the Jacobian operator. Because of the disparity between the horizontal and vertical length scales in the geophysical Ekman layer, EL neglected horizontal diffusion. For completeness we use equal horizontal and vertical diffusivities. Model results with equal horizontal and ver- 
tical diffusivities are found to be virtually identical to those with just vertical diffusion. Finally, Eqs. (7)-(9) have been nondimensionalized using $\Omega_{s}^{-1}$ as the timescale and $K^{1 / 2} \Omega_{s}^{-1 / 2}$ as the length scale.

\section{b. Boundary conditions}

To first approximation, the hurricane boundary layer is a shear-driven turbulent flow. For simplicity, the stress in the surface layer is parameterized with a quadratic drag law whose top resides at $z=0$ and the Ekman layer is parameterized with a constant $K$-theory closure (e.g., Holton 1992, section 5.3.2). ${ }^{2}$ Continuity of stress implies that the two representations of stress must match at the top of the surface layer. Stress continuity together with the condition that the lower boundary is impermeable then gives the following boundary conditions on $z=0$ :

$$
\begin{aligned}
m_{z} & =C_{D} r \tilde{v} \sqrt{u^{2}+\tilde{v}^{2}} \\
\psi_{z z} & =C_{D} \psi_{z} \sqrt{u^{2}+\tilde{v}^{2}} \\
\psi & =0 \\
\eta & =-\frac{1}{r} \psi_{z z}
\end{aligned}
$$

Here and elsewhere $\tilde{v}$ denotes the tangential wind relative to the boundary surfaces. Equations (10) and (11) ensure continuity of stress in the tangential and radial directions at the top of the surface layer, while Eqs. (12) and (13) arise from the impermeability of the lower boundary surface.

The upper boundary $(z=H)$ is assumed stress-free and impermeable:

$$
m_{z}=\psi_{z z}=\psi=\eta=0 .
$$

At the vortex center $(r=0)$ symmetry and regularity require that

$$
m=\psi=\eta=0 .
$$

Finally, the outer boundary $\left(r=r_{\text {out }}\right)$ is assumed impermeable and the outerflow is assumed irrotational:

$$
\psi=m_{r}=0
$$

\section{c. Drag coefficient and eddy diffusivity}

The benchmark experiments in section 4 employ a constant drag coefficient of $2 \times 10^{-3}$. This value is considered reasonable, but uncertainty exists in the actual drag coefficient over the ocean at wind speeds greater than $20 \mathrm{~m} \mathrm{~s}^{-1}$ (e.g., Emanuel 1995). The neutral drag coefficient over the ocean varies from $1.0 \times 10^{-3}$ to 4.0

\footnotetext{
${ }^{2}$ A recent study by Braun and Tao (2000) demonstrates that the bulk-aerodynamic parameterization fairs well against more sophisticated boundary layer schemes in hurricanes.
}

$\times 10^{-3}$ with wind speeds of 5.5 to $52 \mathrm{~m} \mathrm{~s}^{-1}$ (Garratt 1977). Garratt (1977) states that there is little support for using constant drag coefficients over a wide range of wind speeds, as would be found in a hurricane. To address this issue an empirically based wind speed-dependent drag coefficient covering the wide range of wind speeds found in a hurricane is used in section 5.

The vertical diffusion of horizontal momentum by turbulent eddies is represented here by a constant eddy diffusivity of $K=50 \mathrm{~m}^{2} \mathrm{~s}^{-1}$. This value is comparable to the value of $20 \mathrm{~m}^{2} \mathrm{~s}^{-1}$ used by EL. Assuming the validity of a $K$-theory closure, observations of the symmetric angular momentum of Hurricane Norbert (1984) provide an opportunity to estimate the vertical eddy diffusivity. Hurricane Norbert (1984) had a maximum tangential wind speed of $52 \mathrm{~m} \mathrm{~s}^{-1}$ and an RMW of $26 \mathrm{~km}$ (Marks et al. 1992). Neglecting $u^{2}$ compared to $\tilde{v}^{2}$, the dimensional equivalent to (10) yields

$$
K \approx \frac{m^{2} C_{D}}{r m_{z}}
$$

Using the data from Fig. 12f in Marks et al. (1992) and $C_{D}=2 \times 10^{-3}$, we obtain $K \approx 600 \mathrm{~m}^{2} \mathrm{~s}^{-1}$ at a radius of $30 \mathrm{~km}$. We note that the lowest level of observations is $500 \mathrm{~m}$ and the estimate of $K$ is sensitive to the vertical slope of $m$ in the boundary layer, which is likely greater below $500 \mathrm{~m}$. The diffusivities used here are believed to be conservative.

\section{d. Domain, grid spacing, and time step}

With the exception of the Olivia simulation discussed in section 5, all experiments presented have a radial domain of $500 \mathrm{~km}$ and a vertical domain $\mathrm{H}$ of 5,10 , or $15 \mathrm{~km}$ (hereafter H5, H10, H15). Virtually identical results were obtained by halving and doubling the outer domain. For all experiments except the Olivia simulation, the radial grid spacing is $5 \mathrm{~km}$ and the vertical grid spacing is $100 \mathrm{~m}$. These values were determined adequate by doubling the number of grid points in both directions and observing virtually identical results. The time step chosen is $54 \mathrm{~s}$, which is below empirical Courant-Friedrich-Lewy criteria based on the advection and diffusion terms in (7) and (8). The results reported here are believed converged at the present resolution. The half-life time for the Olivia vortex (Fig. 9) has been verified with an entirely different numerical model based on numerical projection operators (D. Nolan 1999, personal communication; see Nolan and Farrell for model details).

\section{Benchmark experiments for tropical storm and hurricane strength vortices}

We first perform benchmark spindown experiments using tropical storm and hurricane strength vortices with fluid depths $\mathrm{H}$ of 5 and $10 \mathrm{~km}$. The tropical storm and hurricane vortices possess Rossby numbers of 40 and 


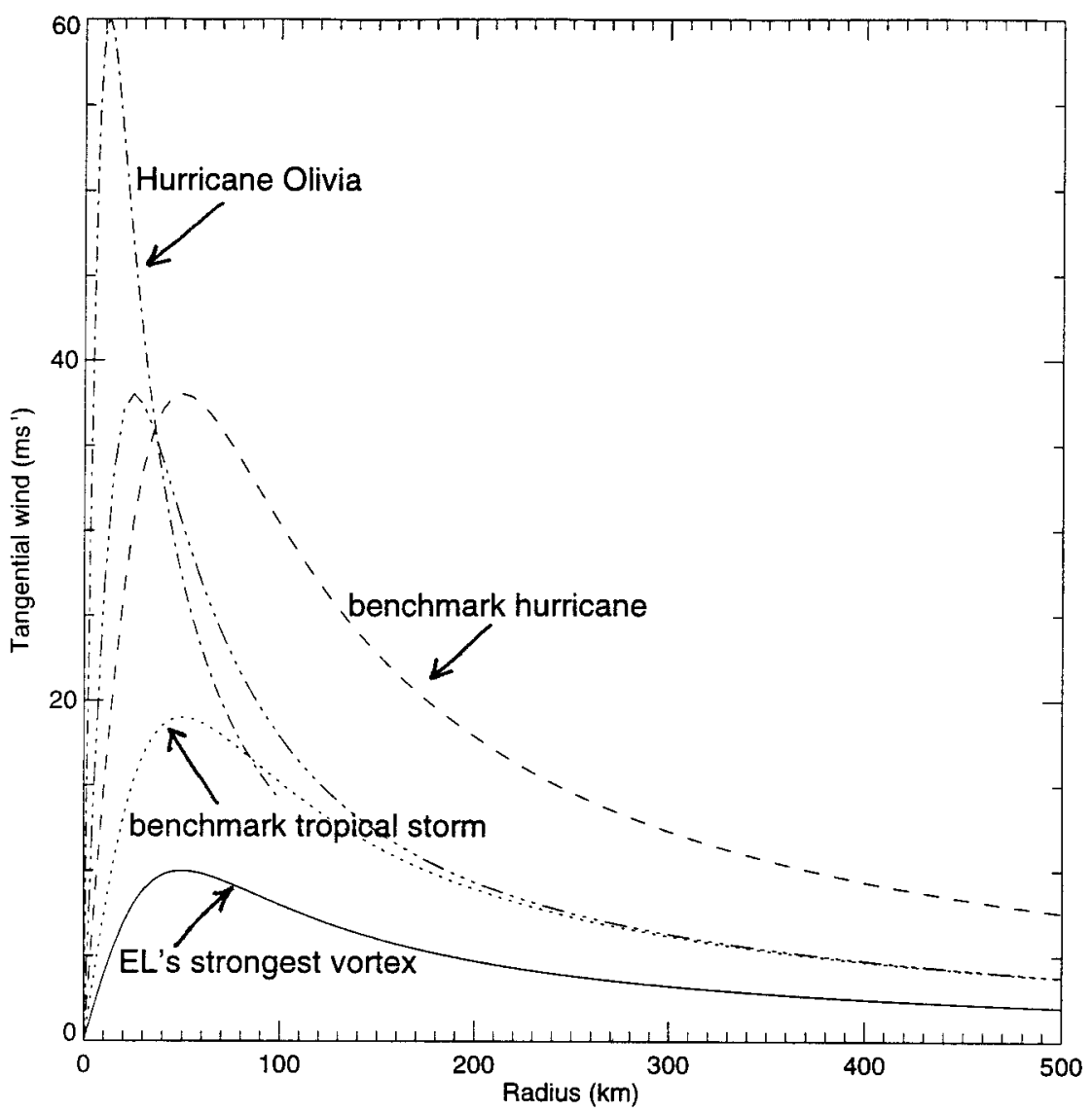

FIG. 2. Initial tangential winds at 1-km height. Shown are strongest vortex used by EL (solid), benchmark tropical storm vortex (dot), and hurricane vortex (dash), and sensitivity cases of Hurricane Olivia simulation (dash-dot) and hurricane vortex with RMW $=25 \mathrm{~km}$ (dash-dotdot-dot).

80, respectively, and RMWs of $50 \mathrm{~km}$. As previously discussed a constant surface drag coefficient $C_{D}=2 \times$ $10^{-3}$ and a constant eddy diffusivity $K=50 \mathrm{~m}^{2} \mathrm{~s}^{-1}$ are employed. Radial profiles of the initial barotropic tangential winds for both benchmark vortices are shown in Fig. 2 along with the other wind profiles that will be considered later. For comparison, EL's strongest vortex is indicated by the solid curve.

Theory and simulations are compared here by evaluating the simulated and theoretical half-life times. The simulated half-life times are calculated by determining the time taken for the tangential winds to decrease to half of their initial value at the RMW. The theoretical half-life time is obtained from equation (6). The initial relative velocity $\tilde{v}$ in (6) is determined by obtaining the maximum tangential winds at $z=1 \mathrm{~km}$ from the model simulations. Based on an examination of model output for the tangential wind, a value of 0.8 is chosen for the reduction factor $\chi$. This value is consistent with hurricane observations (Powell 1980; Black and Franklin 1999, personal communication). Following EL, the vortex boundary layer height $h$ is defined by the height at which the radial wind vanishes at the RMW (i.e., the depth of the inflow layer). In practice, we calculate this depth at the simulated half-life time, although the values are observed to be approximately steady for several halflife times once the boundary layer forms. Strictly speaking, $h$ does not coincide with and is generally greater than the traditional boundary layer depth defined as the $e$-folding scale $h_{m}$ of the angular momentum deficit $M$ $-m$ in the boundary layer, $h_{m}=\sqrt{2 K / I}$, where $I^{2}$ is the inertial (centrifugal) stability of the vortex $r^{-3} \partial M^{2} / \partial r$ (assumed positive throughout). From EL's Fig. 1, the difference between $h_{m}$ and $(h / 1.7)$ increases with increasing Rossby number and obtains a maximum near the RMW for a Rossby number of 20. Consistent with the trends observed by EL, $h$ is found to be $2-3$ times $h_{m}$ for the stronger vortices used here.

\section{a. Tropical storm vortex}

From the model data, the boundary layer depths were determined to be $1 \mathrm{~km}$ for the $H=5 \mathrm{~km}$ case and 1.25 $\mathrm{km}$ for the $H=10 \mathrm{~km}$ case. The predicted half-lifes are then 46 and $100 \mathrm{~h}$ for $\mathrm{H} 5$ and H10, respectively. Since the observed boundary layer height is found to 

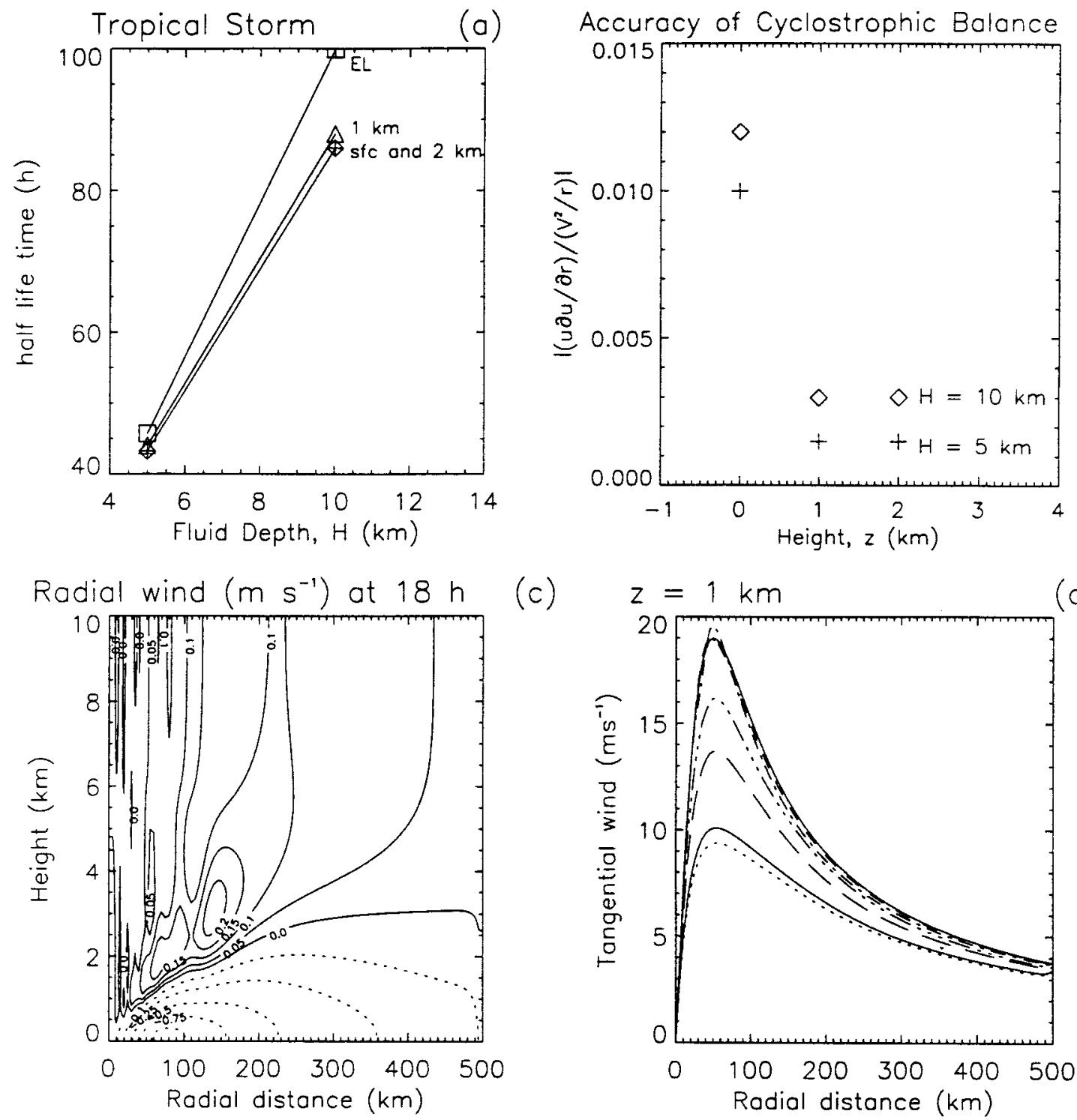

(c)

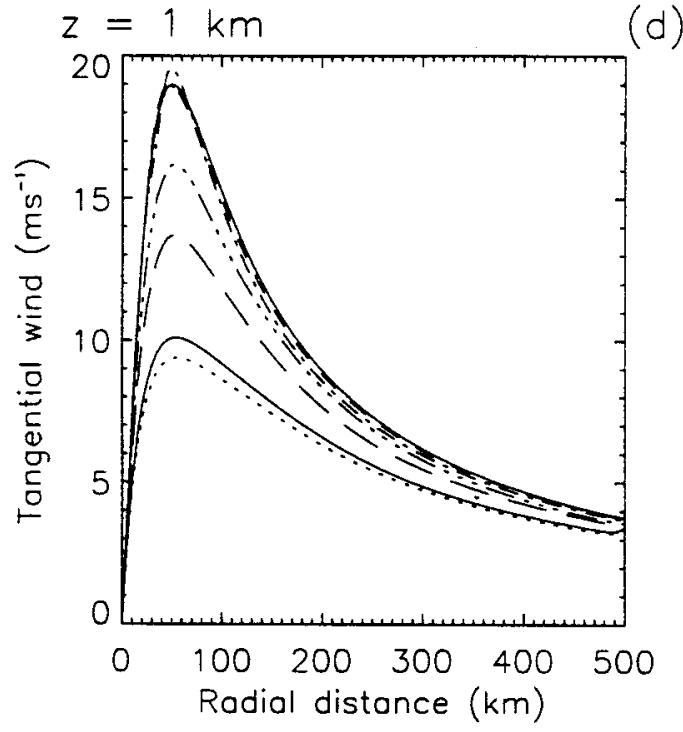

FIG. 3. Tropical storm vortex. (a) Half-life as a function of fluid depth $H$ evaluated at the surface and at 1 and $2 \mathrm{~km}$ above the surface. (b) Assessing the validity of the cyclostrophic balance assumption. Plotted is the maximum absolute ratio of the radial acceleration $u \partial u / \partial r$ to the centripetal acceleration $v^{2} / r$ at the surface, $z=1$ and $2 \mathrm{~km}$. (c) Radial wind as a function of radius and height at $18 \mathrm{~h}$ for the 10-km depth experiment. Outflow denoted by solid lines and inflow by dotted lines. Outflow contour intervals: $0.0,0.05,0.1,0.15$, and $0.2 \mathrm{~m} \mathrm{~s}^{-1}$. Inflow contour intervals: $-0.1,-0.25,-0.5,-0.75,-1.0 \mathrm{~m} \mathrm{~s}^{-1}$. (d) Tangential winds as a function of radius at $z=1 \mathrm{~km}$ for the 10-km depth experiment at 9 min and $78 \mathrm{~h}$ (solid lines), $36 \mathrm{~min}$ and $90 \mathrm{~h}$ (dotted lines), 1.5 $\mathrm{h}$ (short dash), $3 \mathrm{~h}$ (dash-dot), $18 \mathrm{~h}$ (dash-dot-dot-dot), and $36 \mathrm{~h}$ (long dash).

increase slightly as the fluid depth increases, the theoretical half-life for the deeper domain is slightly more than twice that of the shallow domain. From Fig. 3 the simulated and theoretical half-lifes exhibit a small discrepancy with each other but the theory is seen to be qualitatively valid for tropical storm strength vortices.

Figure $3 \mathrm{a}$ indicates that the half-lifes predicted by the model for H5 and H10 are slightly shorter than theory. But as predicted by theory, the half-life approximately doubles with a doubling of the fluid depth. The discrepancy of the simulated half-lifes with theory increas- es with increasing fluid depth. One possible source of discrepancy between the theory and the simulations concerns the validity of the cyclostrophic balance approximation. To determine if the cyclostrophic approximation breaks down, Fig. 3b displays the maximum ratio of the radial acceleration $u \partial u / \partial r$ to the centripetal acceleration $v^{2} / r$ for the $\mathrm{H} 5$ and $\mathrm{H} 10$ experiments at three representative vertical levels in the fluid. The complete radial acceleration $D u / D t$ is found to be approximately twice the magnitude of $u \partial u / \partial r$ for all experiments analyzed. Hence, $u \partial u / \partial r$ is used as a proxy. The ratio 

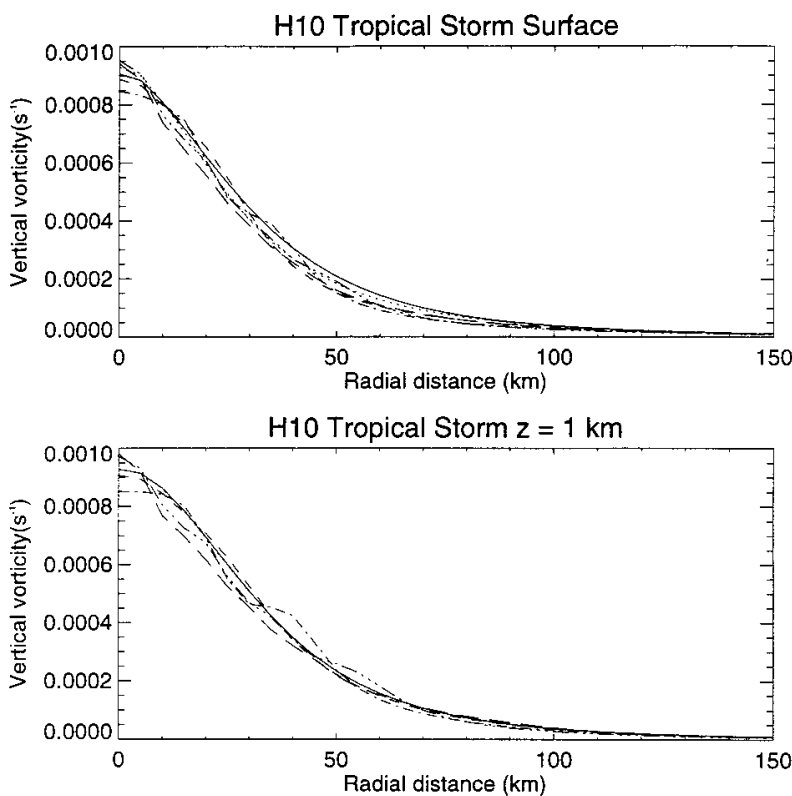

FIG. 4. Vertical vorticity for tropical storm vortex with $H=10 \mathrm{~km}$ at $9 \mathrm{~min}$ (solid line), $36 \mathrm{~min}$ (dotted line), $1.5 \mathrm{~h}$ (short dash), $3 \mathrm{~h}$ (dash-dot), 6 h (dash-dot-dot-dot), and 12 h (long dash).

attains a slightly larger error for the larger fluid depth (e.g., 0.01 for H5 vs 0.012 for H10 at the lower surface). Nevertheless, the cyclostrophic balance approximation appears to be quite accurate for tropical storm strength vortices.

A weak outflow maximum (or jet) just above the boundary layer is evident in Fig. 3c that is not predicted by theory. In the H10 case, the radial outflow jet occurs over several hours. As an example, the radial wind plot in Fig. $3 \mathrm{c}$ at $18 \mathrm{~h}$ reveals a radially broad outflow jet with a maximum radial wind of $0.23 \mathrm{~m} \mathrm{~s}^{-1}$ above $1 \mathrm{~km}$. Radial (inertial) oscillations are evident near the center of the vortex. The outflow jet represents enhanced divergence above the boundary layer which results in a faster spindown. The outflow jet is not evident in the H5 case, which is consistent with the smaller deviation from theory.

A second but not unrelated feature observed in the NS simulations is the spin-up of the tangential winds and vertical vorticity in the boundary layer. Figure $3 d$ shows that at $z=1 \mathrm{~km}$, the local spinup of the tangential winds is $0.75 \mathrm{~m} \mathrm{~s}^{-1}$ at $3 \mathrm{~h}$ for the $\mathrm{H} 10$ case. As the fluid depth increases from $\mathrm{H} 5$ to $\mathrm{H} 10$, the local spinup strengthens and the discrepancy between the simulations and the theory increases. The local spinup of vertical vorticity is highlighted in Fig. 4, which shows the vertical vorticity for the $\mathrm{H} 10$ case as a function of radius at $z=0$ and $1 \mathrm{~km}$. The local spinup is stronger at $z=$ $1 \mathrm{~km}$ than at $z=0$. At $3 \mathrm{~h}$, the vorticity at $z=1 \mathrm{~km}$ increases by $1 \times 10^{-4} \mathrm{~s}^{-1}$ at a radius of $35 \mathrm{~km}$, while at $6 \mathrm{~h}$ the vorticity increases by $6 \times 10^{-5} \mathrm{~s}^{-1}$ at a radius of $55 \mathrm{~km}$.
The origin of the vorticity spinup can be ascertained from the vertical vorticity equation. The vertical vorticity equation for axisymmetric dynamics is given by

$$
\zeta_{t}=-u \zeta_{r}-w \zeta_{z}+w_{z} \zeta-w_{r} v_{z}
$$

where $\zeta=v / r+v_{r}$ is the vertical vorticity, $\zeta_{t}$ the tendency of the vertical vorticity, $-u \zeta_{r}$ the radial advection of vertical vorticity, $-w \zeta_{z}$ the vertical advection of vertical vorticity, $-w_{z} \zeta$ the stretching of vertical vorticity, and $-w_{r} v_{z}$ the tilting term. \{The radial and vertical diffusion terms are not presented here because they are small $\left[O\left(10^{-2}\right)\right]$ compared to the other terms. $\}$ Figure 5 shows these four terms along the surface $(z=0)$ and $z=1 \mathrm{~km}$. On the surface, the tilting and the vertical advection terms are zero since $w=0$ at $z=0$ and convergence dominates over radial advection. Increased convergence in the boundary layer leads to stronger divergence aloft, which leads to a faster spindown. At $z=1 \mathrm{~km}$, all terms contribute to the spinup at different times, but the stretching and tilting terms dominate.

\section{b. Hurricane vortex}

As the vortex strength increases from tropical storm to hurricane strength, the Rossby number increases from 40 to 80 . The observed boundary layer heights for the $\mathrm{H} 5$ and H10 hurricane cases are 800 and $1000 \mathrm{~m}$, respectively. For a wind speed of $38 \mathrm{~m} \mathrm{~s}^{-1}$, the predicted half-life is found to be $24 \mathrm{~h}$ for $\mathrm{H} 5$ and $52 \mathrm{~h}$ for $\mathrm{H} 10$. Since the observed boundary layer height increases slightly with fluid depth, the half-life time for the deeper domain is slightly more than twice that of the shallow domain. As shown by Fig. 6, the theory remains quantitatively valid for the hurricane vortex.

Figure $6 \mathrm{a}$ indicates that the half-life increases with fluid depth and there is a general agreement between theory and simulation. As discussed above, the cyclostrophic approximation is one source of discrepancy between theory and experiment. Figure $6 \mathrm{~b}$ shows that the cyclostrophic approximation becomes marginally valid at the surface but remains valid in the interior for the hurricane strength vortex. The maximum ratio of $u \partial u / \partial r$ to $v^{2} / r$ at the surface in the H10 case is 0.2 , an order of magnitude larger than the H10 tropical storm case.

As in the tropical storm case, the outflow jet is another unpredicted feature. In contrast to the tropical storm case, an outflow jet is evident in the H5 hurricane case (not shown) but with a smaller intensity than in the H10 case. The outflow jet in the H10 hurricane case is more pronounced than in the H10 tropical storm case. Figure $6 \mathrm{c}$ shows an outflow jet centered at approximately 70$\mathrm{km}$ radius with a maximum radial wind of $3.8 \mathrm{~m} \mathrm{~s}^{-1}$ above the boundary layer at $6 \mathrm{~h}$. Radial (inertial) oscillations are also evident near the center of the vortex. As in the tropical storm case, the existence of the enhanced outflow is consistent with a shorter half-life time in the interior flow.

A local spinup of the tangential winds and vertical 

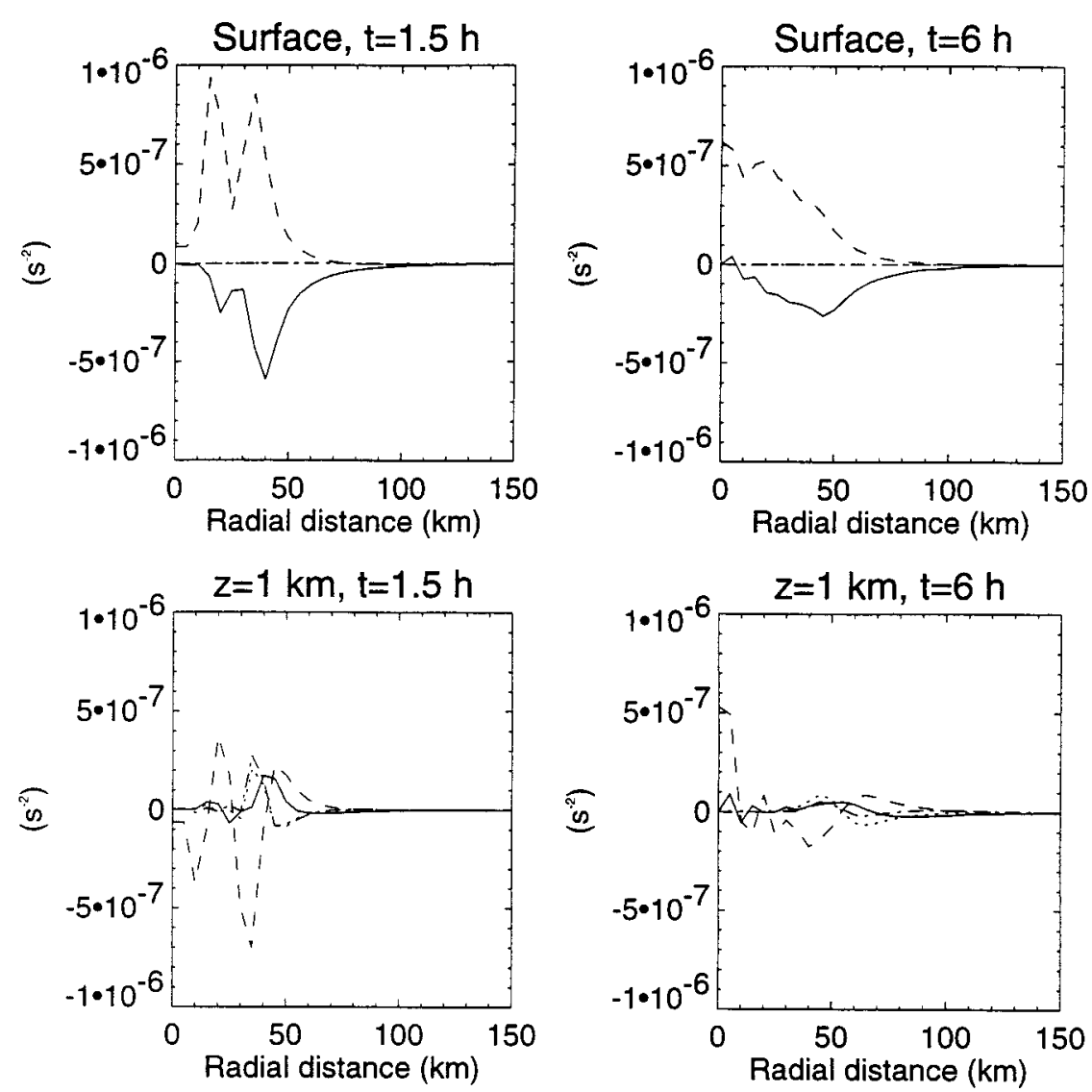

FIG. 5. Vertical vorticity budget for tropical storm vortex with $H=10 \mathrm{~km}$ : radial advection (solid line), vertical advection (dotted line), stretching term (short dash), and tilting term (dashdot). Note that the tilting and vertical advection terms are zero along the lower surface due to $w$ $=0$ at $z=0$.

vorticity is also observed in the hurricane case. A spinup of $0.5 \mathrm{~m} \mathrm{~s}^{-1}$ in the surface tangential winds is observed near $1.5 \mathrm{~h}$ in the H10 case (not shown). Figure $6 \mathrm{~d}$ shows a spinup of $1 \mathrm{~m} \mathrm{~s}^{-1}$ in the maximum tangential winds at 1.5 and $3 \mathrm{~h}$ at $z=1 \mathrm{~km}$. A related spinup occurs in the vertical vorticity and corresponding radial profiles for the $\mathrm{H} 10$ case are shown in Fig. 7. At $1.5 \mathrm{~h}$ on the lower surface, the vorticity increases by $2.5 \times 10^{-4} \mathrm{~s}^{-1}$ at a radius of $30 \mathrm{~km}$. Figure 8 shows that vorticity convergence dominates radial vorticity advection at the lower surface. At $z=1 \mathrm{~km}$, the local spinup is still evident with a magnitude of $5 \times 10^{-4} \mathrm{~s}^{-1}$ at a radius of $40 \mathrm{~km}$. At $z=1 \mathrm{~km}$, the vertical vorticity budget shows that radial, vertical advection, and tilting terms all contribute to the spinup within the RMW.

\section{Sensitivity tests}

In the previous section, the spindown process for tropical storm and hurricane strength vortices was examined and the NS simulations were compared against the theory. Overall, the theory quantitatively captures the spindown physics despite the cyclostrophic balance ap- proximation becoming marginally valid near the surface. According to theory, the half-life is not only proportional to the fluid depth but is also inversely proportional to the drag coefficient and the tangential wind speed. The sensitivity of the model to the maximum tangential wind speed, radius of maximum tangential wind, and constant and variable drag coefficients will now be examined.

\section{a. Dependence on maximum tangential winds}

The sensitivity to the maximum tangential wind speed and the RMW was tested for two cases: an idealized hurricane vortex possessing $38 \mathrm{~m} \mathrm{~s}^{-1}$ maximum tangential winds but with a reduced RMW of $25 \mathrm{~km}$ (Rossby number 160), and an idealized vortex resembling hurricane Olivia (1994; see section 6 for details) that possessed $60 \mathrm{~m} \mathrm{~s}^{-1}$ mean tangential winds and an RMW of $12 \mathrm{~km}$ (Rossby number 526). The RMW $=25 \mathrm{~km}$ case was run with a fluid depth of 10 and $15 \mathrm{~km}$ and the same setup as discussed in section 3. A different model setup is chosen for the Hurricane Olivia simulation because it is smaller and stronger than the other 


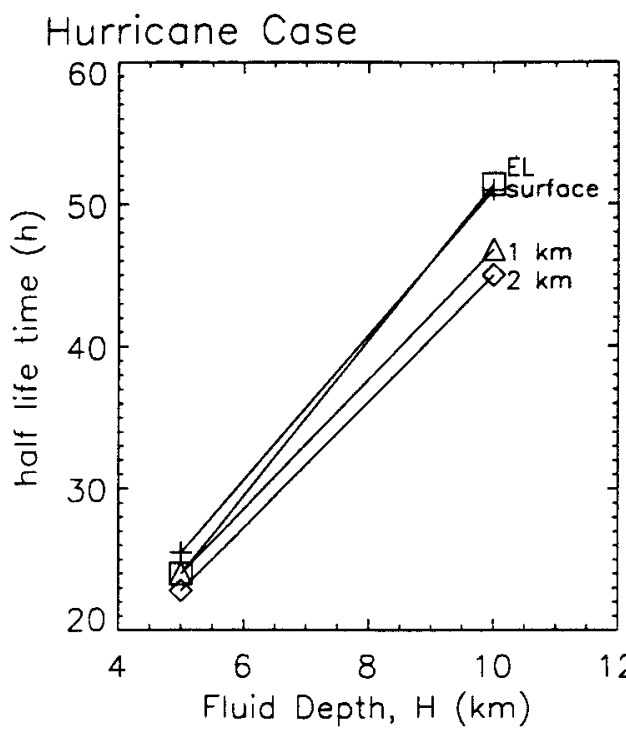

(a)
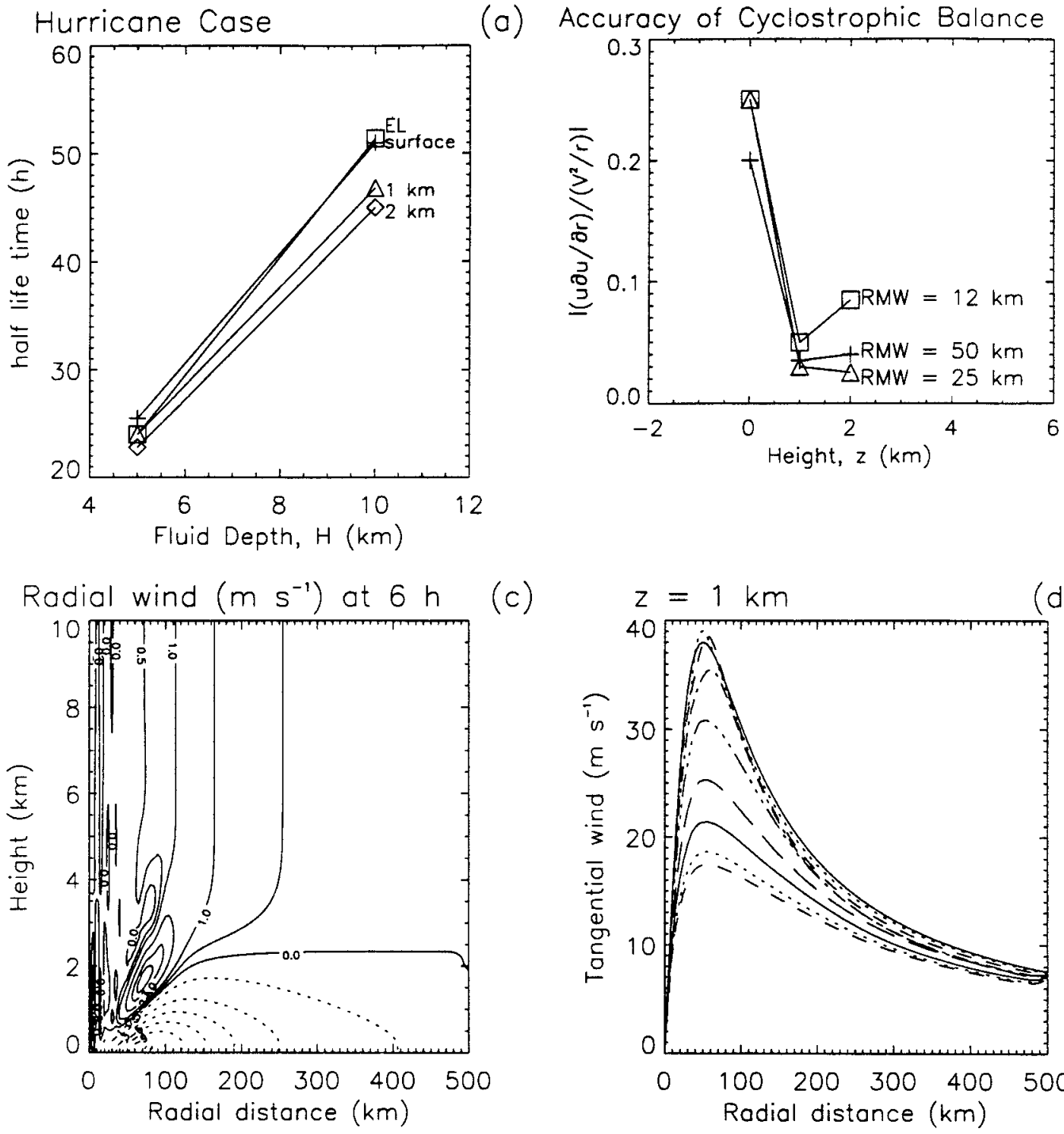

(c)

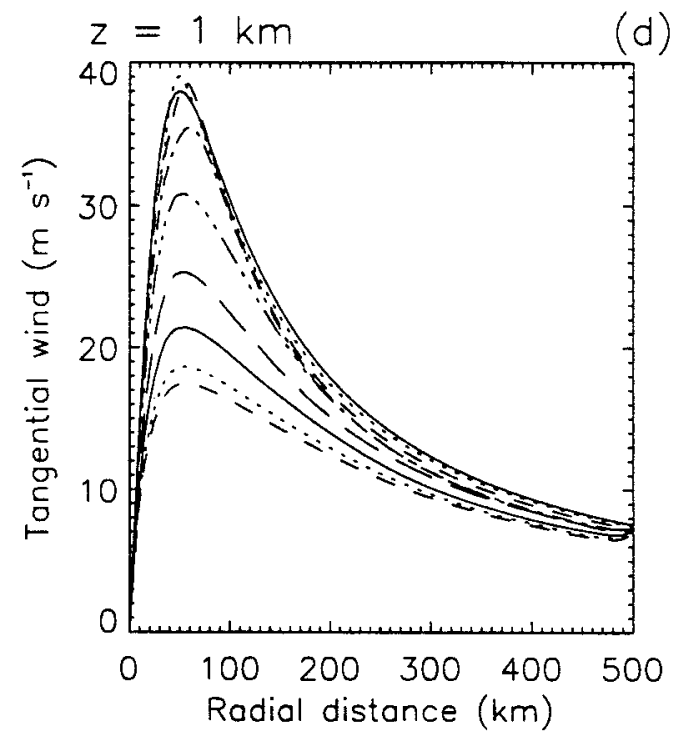

FIG. 6. Hurricane vortex. (a) Hurricane vortex half-life as a function of fluid depth evaluated at the surface and at 1 and $2 \mathrm{~km}$ above the surface. (b) Assessing the validity of the cyclostrophic balance assumption. Plotted is the maximum absolute ratio of the radial acceleration $u \partial u / \partial r$ to the centripetal acceleration $v^{2} / r$ for the hurricane vortex with an RMW of 25 and $50 \mathrm{~km}$, and the Hurricane Olivia simulation vortex with an RMW of $12 \mathrm{~km}$ and a maximum tangential wind speed of $60 \mathrm{~m} \mathrm{~s}^{-1}$. (c) Radial wind for $H=10 \mathrm{~km}, \mathrm{RMW}=50 \mathrm{~km}$ at $6 \mathrm{~h}$. Outflow denoted by solid lines and inflow by dotted lines. Outflow contour intervals: 0.0 , $0.5,1.0,2.0,3.0, \ldots, \mathrm{m} \mathrm{s}^{-1}$. Inflow contour intervals: $-1,-3,-5,-7,-9,-11 \mathrm{~m} \mathrm{~s}^{-1}$. (d) Plot of the tangential winds as a function of radius for $H=10 \mathrm{~km}, \mathrm{RMW}=50 \mathrm{~km}$, and $z=1 \mathrm{~km}$ at 9 min and $36 \mathrm{~h}$ (solid lines), 1.5 and $48 \mathrm{~h}$ (dotted lines), 3 and $54 \mathrm{~h}$ (short dash), $6 \mathrm{~h}$ (dash-dot), $12 \mathrm{~h}$ (dash-dot-dot-dot), and $24 \mathrm{~h}$ (long dash).

vortices considered here. The Olivia simulation has a radial grid spacing of $1 \mathrm{~km}$, a radial domain of $100 \mathrm{~km}$, a time step of $5.4 \mathrm{~s}$, a fluid depth of $10 \mathrm{~km}$, and an eddy diffusivity of $100 \mathrm{~m}^{2} \mathrm{~s}^{-1}$. The boundary layer depths are $500 \mathrm{~m}$ for the Olivia simulation and 750 and 1000 $\mathrm{m}$ for both the $25 \mathrm{~km}$ RMW H10 and H15 cases, respectively. For stronger vortices, the simulations exhibit half-lifes inversely proportional to the maximum tangential wind speed as predicted by the theory. Figure 9 shows the half-lifes as a function of fluid depth (for the 25-km RMW vortex), maximum tangential wind for the H10 cases, RMW for the H10 hurricane cases, and constant drag coefficient for the H5 hurricane case.

Figure 9a shows consistency between the simulations and the theory for an RMW of $25 \mathrm{~km}$. Consistent with the theory, the half-life times for $\mathrm{H} 15$ are approximately 1.5 times the H10 values. As observed in section 4, the quantitative discrepancy between the theoretical and simulated half-life time increases with the fluid depth.

Consistent with theory, Fig. 9b shows that the half- 

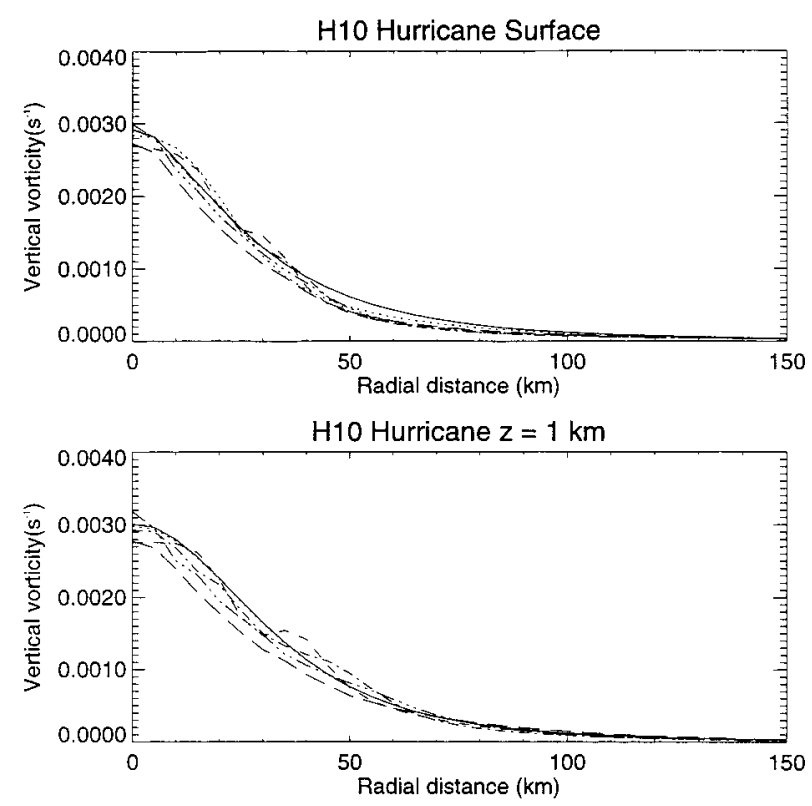

FIG. 7. Vertical vorticity for hurricane vortex with $H=10 \mathrm{~km}$ at 9 min (solid line), 36 min (dotted line), $1.5 \mathrm{~h}$ (short dash), $3 \mathrm{~h}$ (dashdot), 6 h (dash-dot-dot-dot), and 12 h (long dash).
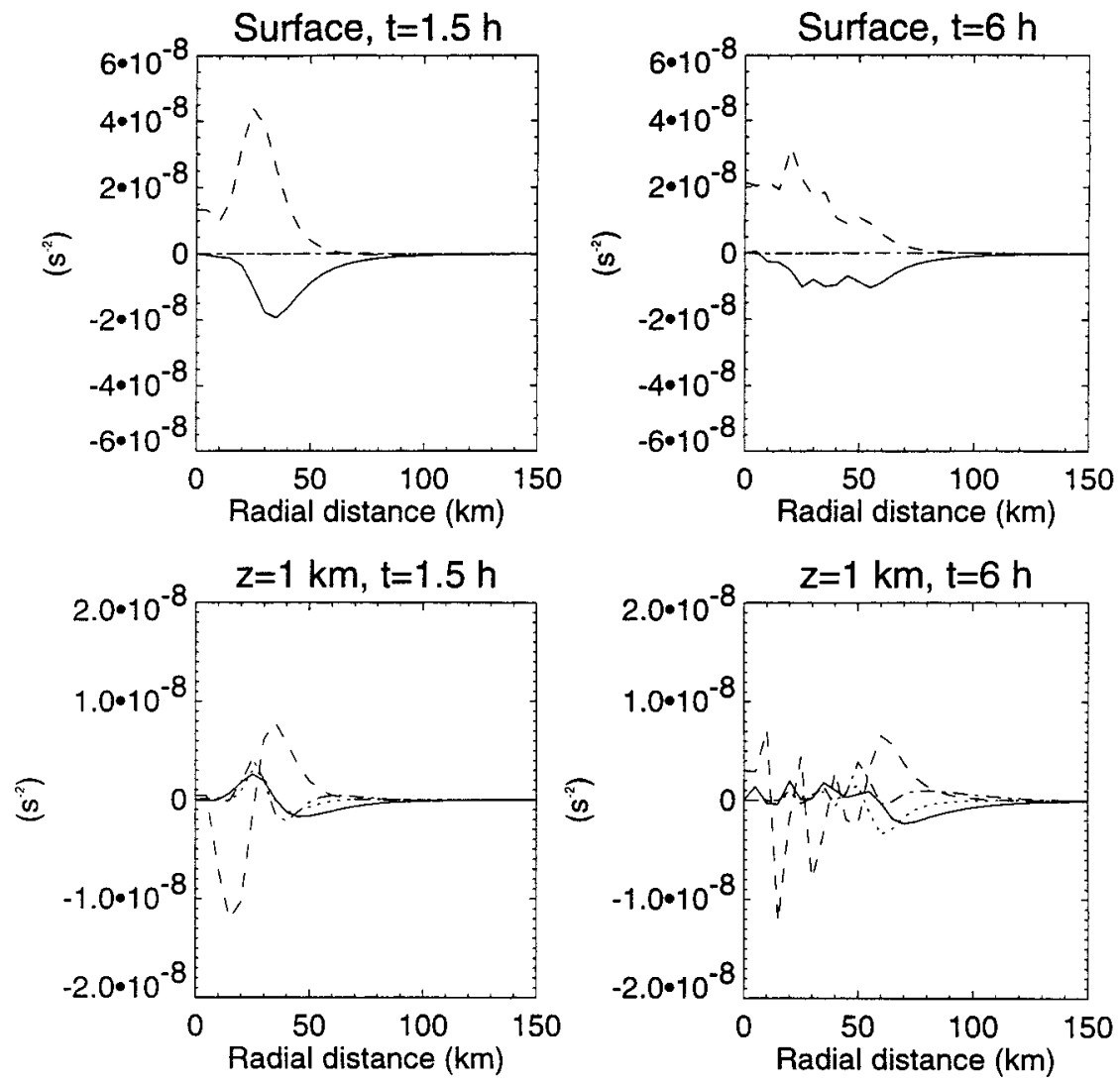

FIG. 8. Vertical vorticity budget for hurricane vortex with $H=10 \mathrm{~km}$ : radial advection (solid line), vertical advection (dotted line), stretching term (short dash), and tilting term (dash-dot). Note that the tilting and vertical advection terms are zero along the lower surface due to $w=0$ at $z=0$. 

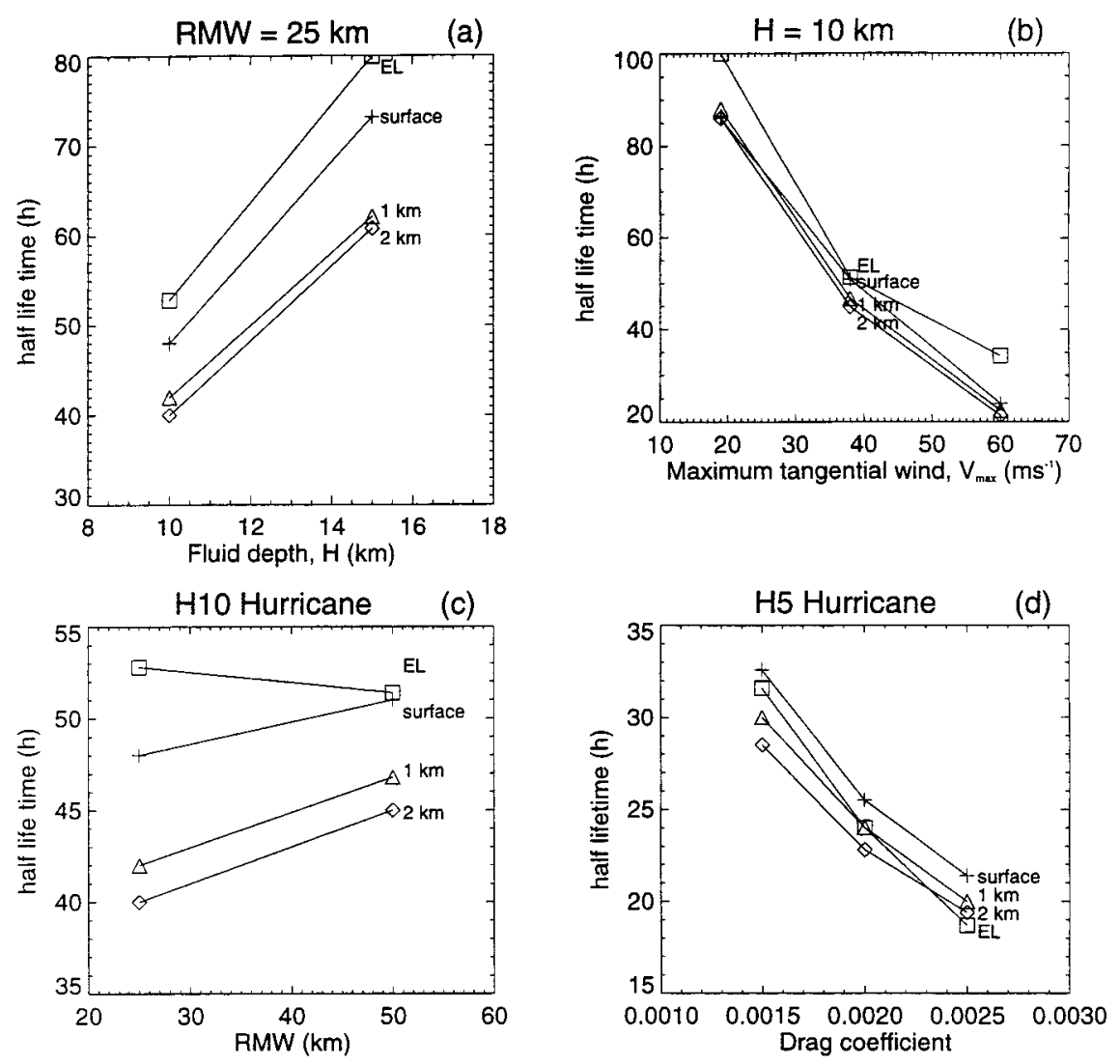

FIG. 9. Vortex half-lifes predicted by the theory and simulations at the surface and at 1 and 2 $\mathrm{km}$, as a function of (a) fluid depth for $H=10$ and $15 \mathrm{~km}$ and RMW $=25 \mathrm{~km}$ hurricane vortex, (b) maximum tangential wind speed for $H=10 \mathrm{~km}$ with half-lifes given for the tropical storm vortex, hurricane vortex, and Hurricane Olivia simulation. (c) Radius of maximum tangential winds for $H=10 \mathrm{~km}$ hurricane vortex, and (d) $H=5 \mathrm{~km}$ hurricane vortex for constant drag coefficients of $1.5 \times 10^{-3}, 2.0 \times 10^{-3}$, and $2.5 \times 10^{-3}$.

and $2.5 \times 10^{-3}$ and a fluid depth of $5 \mathrm{~km}$. The results generalize to other depths by a multiplicative scaling. Figure 9d shows that as the constant drag coefficient increases, the half-life decreases, as expected from theory. The theory has the greatest agreement with a drag coefficient of $2.0 \times 10^{-3}$, which is a value used by EL.

\section{c. Wind speed-dependent drag coefficient}

The wind speed-dependent drag coefficient formulas of Deacon (Roll 1965) and Ooyama (1969) are now used to test the sensitivity of the spindown results for the benchmark hurricane case and Olivia vortex. Deacon's empirical drag formula has been used in many tropical cyclone model simulations (e.g., Rosenthal 1978; Shapiro 1983; Rotunno and Emanuel 1987). Powell (1980), however, found Deacon's formula to give lower values of drag than observed in some hurricanes. As above, Ooyama's and Deacon's formulas are used for the H5 hurricane strength vortex. Deacon's formula (Roll 1965) is

$$
C_{D}=[1.10+(0.04 \mathrm{~V})] \times 10^{-3},
$$

while Ooyama's (1969) formula is

$$
C_{D}=[0.5+(0.06 v)] \times 10^{-3},
$$

In (19) and (20), the winds are assumed to be at a height of $10 \mathrm{~m}$ and are in units of $\mathrm{m} \mathrm{s}^{-1}$. The total horizontal wind speed is used in Deacon's formula while only the tangential wind is used in Ooyama's formula.

For these experiments, the theoretical half-life times are obtained from the simulated drag coefficient at the RMW and the simulated maximum tangential wind speed at $z=1 \mathrm{~km}$. As before, a reduction factor of 0.8 is used. The boundary layer depth and $\tilde{v}$ are determined by the method described earlier. Figure 10 summarizes the simulated half-life times for these variable drag coefficient experiments including the dependence on the fluid depth, RMW, and maximum tangential wind.

From Fig. 10a, the simulated half-life time at $2 \mathrm{~km}$ is closest to theory for both wind speed-dependent drag coefficients. The largest discrepancies with theory occurs at the surface. Based on the preceding results the discrepancy is associated with the deterioration of the cyclostrophic approximation in the boundary layer. Due 

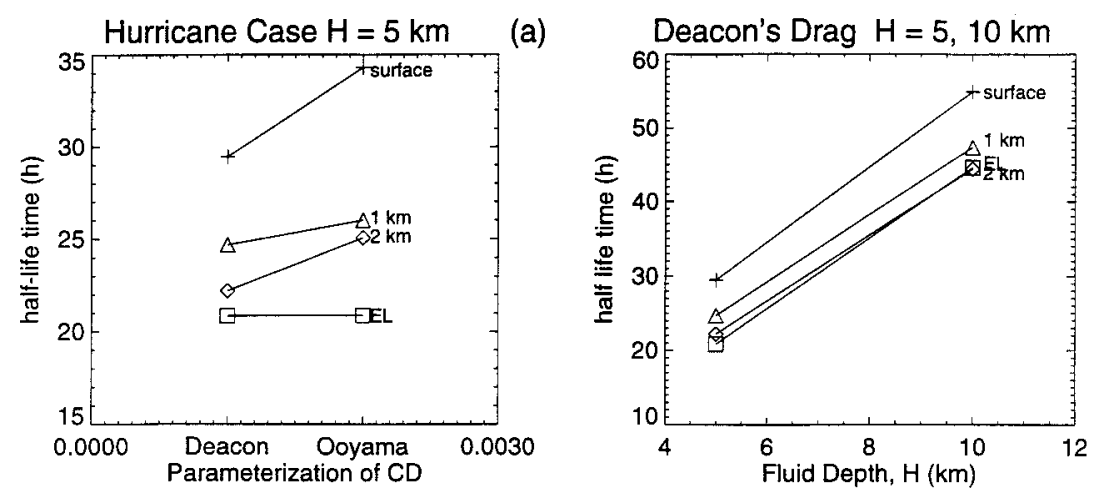

(b)

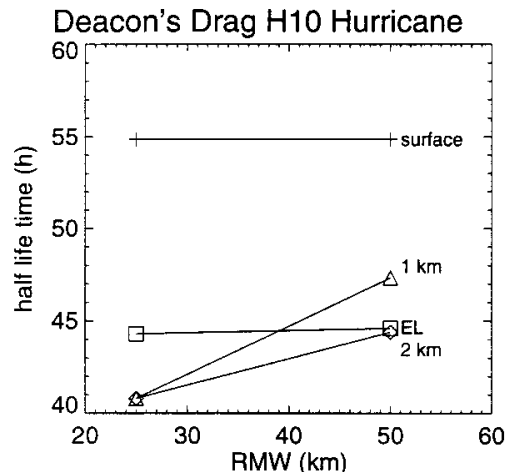

(c)

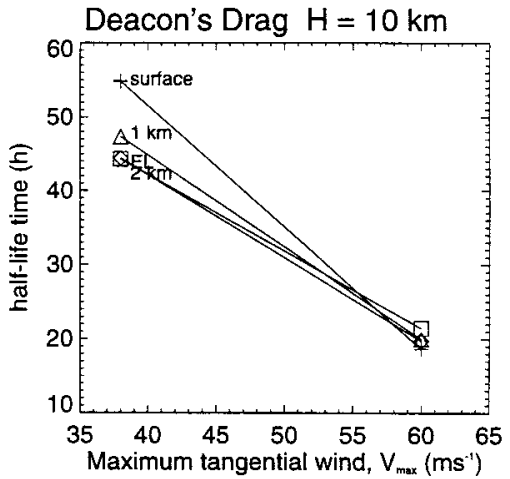

(d)

FIG. 10. Vortex half-lifes predicted by the theory and simulations at the surface and at 1 and $2 \mathrm{~km}$, as a function of (a) Deacon's or Ooyama's variable drag coefficient for $H=5 \mathrm{~km}$ hurricane vortex, (b) the $H=5$ and $10 \mathrm{~km}$ hurricane vortex using Deacon's formula, (c) radius of maximum tangential winds for $H=10 \mathrm{~km}$ hurricane case using Deacon's formula, and (d) maximum tangential wind speed for $H=10 \mathrm{~km}$ using Deacon's formula with half-life times given for the tropical storm vortex, hurricane vortex, and Hurricane Olivia simulation.

to a better correspondence with the theory, Deacon's formula is used for the remaining experiments shown in Figs. 10b,c,d.

Figure 10b shows that the theory remains quantitatively valid for different fluid depths with a wind speeddependent drag coefficient. With variable drag the discrepancy found previously with increasing fluid depth diminishes above the boundary layer. As the fluid depth is doubled to $H=10 \mathrm{~km}$, the half-life at $z=2 \mathrm{~km}$ exactly doubles as predicted by theory. At the surface and $z=1 \mathrm{~km}$, the half-lifes are slightly less than double. When the RMW is decreased from 50 to $25 \mathrm{~km}$ and all other variables are kept constant, Fig. 10c shows that the theory agrees well for the interior flow while it is slightly offset for the near-surface flow.

The Olivia simulation with Deacon's wind speeddependent drag formula is the most realistic case presented here. The results in Fig. 10d demonstrate that the theory remains quantitatively valid for realistic hurricane-like vortices. The simulated half-lifes are much closer to theory with a variable drag coefficient than with a constant drag coefficient (cf. Fig. 9b). The best compatibility of the simulations with theory is in the interior flow where the cyclostrophic balance assumption remains valid for stronger vortices.

In summary, EL's theory has been shown to hold valid for realistic hurricane-like vortices for constant and variable drag coefficients, different fluid depths, tangential wind speeds, and RMWs.

\section{Comparison between theory and observations}

Eliassen and Lystad's theory has been shown to apply to hurricane strength vortices, but can EL's theory help explain observed hurricane behavior? Let us now investigate the applicability of EL's theory to observations obtained by research aircraft during the weakening phase of Hurricanes Allen (1980) and Olivia (1994). Using the initial observed tangential winds for these vortices, a comparison is made between the predicted and observed tangential winds at a later time. The interior rotation rate is predicted from (5) and the tangential wind is obtained by multiplying $\Omega$ by $r$.

In this comparison the fluid depth $H$ is chosen to be $15 \mathrm{~km}$. As in preceding sections a reduction factor of $\chi=0.8$ is employed. Constant drag coefficients of 2 
$\times 10^{-3}, 2.5 \times 10^{-3}$, and $3.0 \times 10^{-3}$ are chosen as well as Deacon's variable drag formula. The near-surface wind for the variable drag formula is obtained by multiplying the initial observed mean tangential winds by $\chi$. If the atmosphere were stably stratified instead of neutrally stratified as assumed, the spindown process would be somewhat slower than predicted here. Although this analysis is dependent on the chosen $H, \chi$, $C_{D}$ in (5) and the assumption that the atmosphere is neutrally stratified, it will nevertheless be shown to furnish a consistent description of the observed behavior.

\section{a. Hurricane Allen (1980)}

Hurricane Allen was a Cape Verde storm that entered the Caribbean as an intense hurricane and underwent dramatic intensification and weakening cycles on the order of 40-50 mb per day (Fig. 10 of Willoughby et al. 1982). Hurricane Allen (1980) experienced a secondary eyewall cycle, which begins with an eyewall forming outside the initial eyewall. The initial eyewall decays in part due to subsidence and low-level outflow from the outer eyewall (Willoughby 1988). The outer eyewall then contracts and finally becomes the primary eyewall. On 8 August 1980 Allen was deepening and the eye was contracting as multiple research aircraft flew through the storm. On 9 August 1980 a single aircraft investigated the weakening eyewall and outer wind maximum as the storm made landfall. During this time Hurricane Allen was filling from approximately 910 to $940 \mathrm{mb}$ and weakening. Estimates of the azimuthal mean tangential wind as a function of radius from the research aircraft data of these missions reported by Willoughby et al. (1982) will be used to test the theory. Specifically, the theory will be tested with the tangential winds at $850 \mathrm{mb}$ at 2030 UTC 8 August and at $700 \mathrm{mb}$ at 2031 UTC 9 August.

From Fig. 14 of Willoughby et al. (1982), the mean tangential wind at $850 \mathrm{mb}$ was approximately $58 \mathrm{~m} \mathrm{~s}^{-1}$ at a radius of $18 \mathrm{~km}$ at 2030 UTC 8 August. By $24 \mathrm{~h}$ later, the maximum mean tangential wind decreased to $42 \mathrm{~m} \mathrm{~s}^{-1}$ at $700 \mathrm{mb}$ according to Fig. 15 of Willoughby et al. (1982). In accord with the theory, we will assume there is no significant variation in the tangential wind between 850 and $700 \mathrm{mb}$ (i.e., a barotropic vortex between these levels). From this data, we obtain an initial rotation rate of $\Omega_{0}=\tilde{v} / r=3.22 \times 10^{-3} \mathrm{~s}^{-1}$. Assuming a neutrally stratified atmosphere with $H \gg h$ and using $\chi=0.8, t=24 \mathrm{~h}$, and $H=15 \mathrm{~km}$ and drag coefficients of $2.0 \times 10^{-3}, 2.5 \times 10^{-3}$, and $3.0 \times 10^{-3}$, the theory predicts maximum tangential winds of 40.6, 37.8, and $35.2 \mathrm{~m} \mathrm{~s}^{-1}$, respectively, after $24 \mathrm{~h}$. The theoretical predictions of the tangential winds are somewhat weaker than those observed in Hurricane Allen. For a drag coefficient of $2.0 \times 10^{-3}$, the difference between the observations and theory is only $1.4 \mathrm{~m} \mathrm{~s}^{-1}$. For an estimated near-surface initial wind speed of $46.4 \mathrm{~m} \mathrm{~s}^{-1}$, we obtain a drag coefficient of $2.956 \times 10^{-3}$ using Deacon's for-

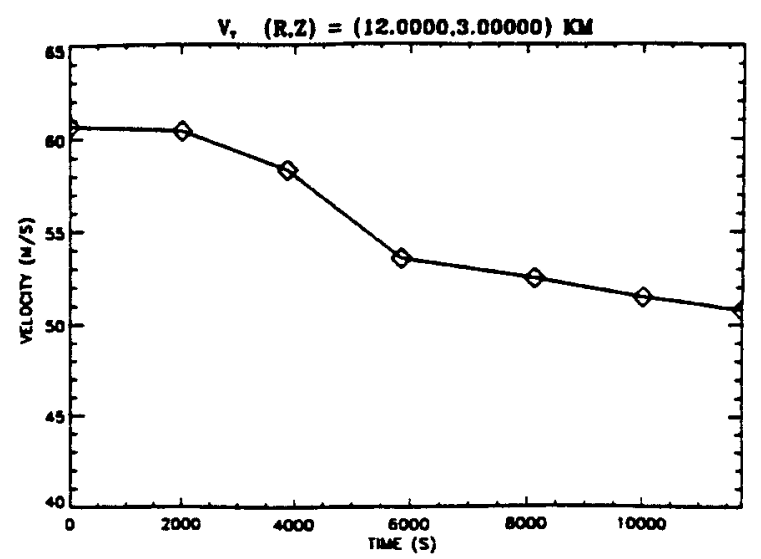

FIG. 11. Hurricane Olivia's (1994) tangential wind over $3.5 h$ at $z$ $=3 \mathrm{~km}$ and a radius of $12 \mathrm{~km}$ on 25 Sep 1994 (Courtesy of P. Reasor).

mula. Substituting this into (5), the predicted wind speed $24 \mathrm{~h}$ later is $35.52 \mathrm{~m} \mathrm{~s}^{-1}$.

\section{b. Hurricane Olivia (1994)}

Tropical depression Olivia formed off the southern tip of Baja California at 0600 UTC 22 September. Olivia became a hurricane with maximum tangential wind speeds of $33 \mathrm{~m} \mathrm{~s}^{-1}$ at $0600 \mathrm{Z} 24$ September and continued to intensify to $67 \mathrm{~m} \mathrm{~s}^{-1}$ at 1200 UTC 25 September. Olivia subsequently came under the influence of a midto upper-tropospheric cyclone that advected it northnortheast at $5 \mathrm{~m} \mathrm{~s}^{-1}$ as it slowly weakened (Pasch and Mayfield 1996). During this time, Hurricane Olivia encountered increasing vertical shear from 5 to $15 \mathrm{~m} \mathrm{~s}^{-1}$ making it more susceptible to frictional effects (Reasor et al. 2000).

During a 3.5-h period on 25 September 1994, Hurricane Olivia weakened from an initial tangential wind speed of $61 \mathrm{~m} \mathrm{~s}^{-1}$ down to $51 \mathrm{~m} \mathrm{~s}^{-1}$ (see Fig. 11) at a height of $3 \mathrm{~km}$. From aircraft observations of Hurricane Olivia, on 25 September 1994, the height of hurricane force winds decreased from approximately 15 to $10 \mathrm{~km}$ in $3.5 \mathrm{~h}$ (P. Reasor 1999, personal communication). Thus, our fluid depth of $15 \mathrm{~km}$ is considered reasonable. With an initial tangential wind of $61 \mathrm{~m} \mathrm{~s}^{-1}$ at a radius of $12 \mathrm{~km}$, we obtain an initial rotation rate of $\Omega_{0}=\tilde{v} / r$ $=5.08 \times 10^{-3} \mathrm{~s}^{-1}$. After $3.5 \mathrm{~h}$, the theory predicts a weakening of the tangential winds to 57.2, 56.3, and $55.5 \mathrm{~m} \mathrm{~s}^{-1}$ for constant drag coefficients of $2.0 \times 10^{-3}$, $2.5 \times 10^{-3}$, and $3.0 \times 10^{-3}$, respectively. In contrast to the Allen case, the theory predicts somewhat stronger tangential winds than observed. Thus, Hurricane Olivia is spinning down faster than predicted by the simple theory for a fluid depth of $15 \mathrm{~km}$. The predicted tangential wind speed of $55.5 \mathrm{~m} \mathrm{~s}^{-1}$ is the closest to the observed value of $51 \mathrm{~m} \mathrm{~s}^{-1}$. With an estimated nearsurface initial wind speed of $48.8 \mathrm{~m} \mathrm{~s}^{-1}$, we obtain a drag coefficient of $3.052 \times 10^{-3}$ using Deacon's for- 
mula. The theory then predicts a corresponding interior tangential wind speed of $55.42 \mathrm{~m} \mathrm{~s}^{-1}$, slightly closer to the observed.

Hurricane Allen (1980) was more symmetric than Hurricane Olivia (1994). And while Allen was experiencing a secondary eyewall cycle the convection in the inner eyewall was strongly suppressed. On the other hand, the vertical shear in Olivia did not suppress the convection but made it more asymmetric. The effects of vertical shear and convection may contribute to the differences observed in the two cases. Given the uncertainties in $\chi, C_{D}$, and $H$ and the assumption that the atmosphere is neutrally stratified, the theory still furnishes consistent estimates.

\section{Conclusions}

Using the approximate cyclostrophic spindown theory of Eliassen and Lystad (1977) for guidance, axisymmetric spindown for tropical cyclone-scale vortices subject to a quadratic drag law in the surface layer has been investigated using a Navier-Stokes numerical model. The numerical model incorporates the noncyclostrophic terms neglected in EL's theory and allows one to examine the consequences of the deterioration of cyclostrophic balance approximation as the initial vortex intensity is increased to hurricane strength and beyond.

The theoretically predicted algebraic temporal decay of the primary flow is measured here by the half-life at the RMW and is validated for tropical storm and hurricane strength vortices. The departure from theory tends to increase with increasing fluid depth but is still qualitatively valid for hurricane-like vortices 10 and 15 $\mathrm{km}$ deep. As the vortex strength increases from tropical storm to hurricane strength, the cyclostrophic balance approximation becomes marginally valid in the boundary layer yet remains valid in the flow interior. A temporary spinup of tangential winds and vertical vorticity in the boundary layer and a low-level outflow jet are observed in the numerical simulations but are not predicted by the theory. These features are argued to be the primary cause for the discrepancy between the theory and the model simulations.

Sensitivity tests of the spindown process to the initial maximum tangential wind, RMW, and drag coefficient reveal that the theory remains qualitatively valid for hurricane strength vortices. Holding the intensity constant, the departure from the theory increases somewhat as the RMW decreases. The theory also holds true for constant drag coefficients ranging from 0.0015 to 0.0025 . Wind speed-dependent drag coefficient simulations agree more closely with the theory than their constant coefficient counterparts. Indeed, the Hurricane Olivia simulation with Deacon's drag formulation gave results remarkably close to theory.

Eliassen and Lystad's theory was found to be qualitatively consistent with aircraft observations of the axi- symmetric evolution of Hurricanes Allen (1980) (Willoughby et al. 1982) and Olivia (1994) (Reasor et al. 2000). Comparing the observations of Hurricane Allen (1980) with theory, Ekman spindown processes help explain the weakening of the inner eyewall of Allen and offer useful insight into the weakening phases of other hurricanes experiencing secondary eyewall replacements.

In summary EL's theory appears useful in elucidating spindown of a geophysical vortex with a turbulent boundary layer, such as a hurricane, when penetrative convection is weak or absent. Future work should consider the effects of stable stratification on the spindown process.

Acknowledgments. This research was supported in part by ONR-N00014-93-1-0456, NOAA NA67RJ0152 Amendment 19, and Colorado State University. This paper is dedicated in memory of Professor Arnt Eliassen. The first author would like to thank Professor Eliassen for inspiring this study, which was reinvigorated during a conversation with him over cocktails at the Generations Symposium at MIT's Endicott House (1995) in honor of Arnt's 80th birthday.

\section{APPENDIX}

\section{Numerical Details}

The numerical method for solving the axisymmetric Navier-Stokes system described in section 3 is summarized here. The method uses finite-difference approximations in both time and space variables. Equations (7) and (8) are discretized using a Crank-Nicholson scheme, an Arakawa Jacobian scheme, and a thirdorder Adams-Bashforth scheme. An implicit CrankNicolson scheme is used in the implementation of vertical diffusion. The Jacobian terms $J(\psi, m)$ and $J(\psi, \eta)$ are evaluated with Arakawa's Jacobian scheme. Upwinding is used for advecting angular momentum on the lower surface $z=0$. The third-order Adams-Bashforth scheme is used for time stepping the Jacobian terms and the radial diffusion of $m$ and $\eta$. Ghost points that arise in solving for $m$ at the upper and lower boundaries are obtained by lagging the surface stress in the square root term of Eq. (10) at the lower boundary. Lagging of the surface stress generally causes computational instability at high swirl speeds, but this is readily eliminated by iterating $m$ to convergence at each radial grid point within each time step. The two predictive equations for $m$ and $\eta$ are solved by a standard tridiagonal solver. The Poisson problem for $\psi$, given $\eta$, is solved via a fast-Poisson solver (Swarztrauber and Sweet 1975). The numerical model predicts $m, \eta$, and $\psi$ fields. The remaining quantities, such as $\tilde{v}, \Omega, u$, and $w$, are then deduced from the output fields. 


\section{REFERENCES}

Braun, S. A., and W.-K. Tao, 2000: Sensitivity of high-resolution simulations of Hurricane Bob (1991) to planetary boundary layer parameterizations. Mon. Wea. Rev., 128, 3941-3961.

Carrier, G. F., 1971: Swirling flow boundary layers. J. Fluid Mech., 49, 133-144.

Dolzhanskii, F. V., V. A. Drymov, and D. Yu. Manin, 1992: Selfsimilar spin-up and spin-down in a cylinder of small ratio of height to diameter. J. Fluid Mech., 234, 473-486.

Eliassen, A., 1971: On the Ekman Layer in a circular vortex. $J$. Meteor. Soc. Japan, 49, 784-789.

—, and M. Lystad, 1977: The Ekman layer of a circular vortex: A numerical and theoretical study. Geophys. Norv., 31, 1-16.

Emanuel, K. A., 1995: Sensitivity of tropical cyclones to surface exchange coefficients and a revised steady-state model incorporating eye dynamics. J. Atmos. Sci., 52, 3969-3976.

Garratt, J. R., 1977: Review of drag coefficients over oceans and continents. Mon. Wea. Rev., 105, 915-929.

Greenspan, H. P., and L. N. Howard, 1963: On a time-dependent motion of a rotating fluid. J. Fluid Mech., 17, 385-404.

Holton, J. R., 1992: An Introduction to Dynamic Meteorology. 3d ed. Academic Press, 507 pp.

Jones, S. C., and C. Thorncroft, 2000: The longevity of tropicalcyclone vortices after extratropical transitions. Preprints, 24th Conf. on Hurricanes and Tropical Meteorology, Fort Lauderdale, FL, Amer. Meteor. Soc., 318-319.

Kaplan, J., and M. DeMaria, 1995: A simple empirical model for predicting the decay of tropical cyclone winds after landfall. $J$. Appl. Meteor., 34, 2499-2512.

Kloosterziel, R. C., and G. J. F. van Heijst, 1992: The evolution of stable barotropic vortices in a rotating free-surface fluid. J. Fluid Mech., 239, 607-629.

Kurihara, Y., 1985: Numerical modeling of tropical cyclones. Advances in Geophysics, Vol. 28B, Academic Press, 255-279.

Marks, F. D., Jr., R. A. Houze Jr., and J. F. Gamache, 1992: Dualaircraft investigation of the inner core of Hurricane Norbert. Part I: Kinematic structure. J. Atmos. Sci., 49, 919-942.

McWilliams, J. C., 1971: The boundary layer dynamics of symmetric vortices. Ph.D. thesis, Harvard University, 89 pp. [Available from University Microfilm, Inc., Ann Arbor, MI 48104.]

Nolan, D. S., and B. F. Farrell, 1999: The structure and dynamics of tornado-like vortices. J. Atmos. Sci., 56, 2908-2936.

Ooyama, K., 1969: Numerical simulation of the life cycle of tropical cyclones. J. Atmos. Sci., 26, 3-40.

Pasch, R. J., and M. Mayfield, 1996: Eastern North Pacific hurricane season of 1994. Mon. Wea. Rev., 124, 1579-1590.

Powell, M. D., 1980: Evaluations of diagnostic marine boundarylayer models applied to hurricanes. Mon. Wea. Rev., 108, 757766.

Reasor, P. D., M. T. Montgomery, F. D. Marks Jr., and J. F. Gamache, 2000: Low-wavenumber structure and evolution of the hurricane inner core observed by airborne dual-Doppler radar. Mon. Wea. Rev., 128, 1653-1680.

Roll, H. U., 1965: Physics of the Marine Atmosphere. International Geophysical Series, Vol. 7, Academic Press, 426 pp.

Rosenthal, S. L., 1962: A theoretical analysis of the field of motion in the hurricane boundary layer. National Hurricane Research Project Rep. 56, U.S. Dept. of Commerce, 12 pp. [Available from NOAA/AOML, Hurricane Research Division, Miami, FL 33149-1026.]

, 1978: Numerical simulation of tropical cyclone development with latent heat release by the resolvable scales I: Model description and preliminary results. J. Atmos. Sci., 35, 258-271.

Rotunno, R., and K. A. Emanuel, 1987: An air-sea interaction theory for tropical cyclones. Part II: Evolutionary study using a nonhydrostatic axisymmetric numerical model. J. Atmos. Sci., 44, 542-561.

Shapiro, L. J., 1983: The asymmetric boundary layer flow under a translating hurricane. J. Atmos. Sci., 40, 1984-1998.

- and M. T. Montgomery, 1993: A three-dimensional balance theory for rapidly rotating vortices. J. Atmos. Sci., 50, 3322 3335 .

Smith, R. K., 1968: The surface boundary layer of a hurricane. Tellus, 20, 473-484.

Swarztrauber, P., and R. A. Sweet, 1975: Efficient FORTRAN subprograms for the solution of elliptic partial differential equations. NCAR Tech. Note TN/IA-109, 139 pp.

Tuleya, R. E., M. A. Bender, and Y. Kurihara, 1984: A simulation study of the landfall of tropical cyclones using a moveable nested-mesh model. Mon. Wea. Rev., 112, 124-136.

Wakimoto, R. M., and P. G. Black, 1994: Damage survey of Hurricane Andrew and its relationship to the eyewall. Bull. Amer. Meteor. Soc., 75, 189-200

Weidman, P. D., 1976: On the spin-up and spin-down of a rotating fluid. Part 1. Extending the Wedemeyer model. J. Fluid Mech., 77, 685-708.

Willoughby, H. E., 1979: Forced secondary circulations in hurricanes. J. Geophys. Res., 84, 3173-3183.

, 1988: The dynamics of the tropical cyclone core. Aust. Meteor. Mag., 36, 183-191.

_ of a disaster. Bull. Amer. Meteor. Soc., 77, 543-549.

_ J. A. Clos, and M. G. Shoreibah, 1982: Concentric eye walls, secondary wind maxima, and the evolution of the hurricane vortex. J. Atmos. Sci., 39, 395-411.

- , H. Jin, S. J. Lord, and J. M. Piotrowicz, 1984: Hurricane structure and evolution as simulated by an axisymmetric, nonhydrostatic numerical model. J. Atmos. Sci., 41, 1169-1186.

Wu, R., and W. Blumen, 1982: An analysis of Ekman boundary layer dynamics incorporating the geostrophic momentum approximation. J. Atmos. Sci., 39, 1774-1782.

Wurman, J., and J. Winslow, 1998: Intense sub-kilometer-scale boundary layer rolls observed in Hurricane Fran. Science, 280, 555-557. 